\title{
Nanoscale
}

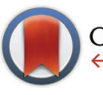

CrossMark

$\leftarrow$ click for updates

Cite this: Nanoscale, 2015, 7, 9545

\section{Surface- and tip-enhanced Raman spectroscopy reveals spin-waves in iron oxide nanoparticles $\uparrow$}

\author{
Raul D. Rodriguez, ${ }^{a, b}$ Evgeniya Sheremet, ${ }^{a}$ Tanja Deckert-Gaudig, ${ }^{c}$ \\ Corinne Chaneac, ${ }^{d}$ Michael Hietschold, ${ }^{a}$ Volker Deckert ${ }^{c, e}$ and Dietrich R. T. Zahn ${ }^{\text {a,b }}$
}

Nanomaterials have the remarkable characteristic of displaying physical properties different from their bulk counterparts. An additional degree of complexity and functionality arises when oxide nanoparticles interact with metallic nanostructures. In this context the Raman spectra due to plasmonic enhancement of iron oxide nanocrystals are here reported showing the activation of spin-waves. Iron oxide nanoparticles on gold and silver tips are found to display a band around $1584 \mathrm{~cm}^{-1}$ attributed to a spin-wave magnon mode. This magnon mode is not observed for nanoparticles deposited on silicon (111) or on glass substrates. Metal-nanoparticle interaction and the strongly localized electromagnetic field contribute to the appearance of this mode. The localized excitation that generates this mode is confirmed by tip-enhanced Raman spectroscopy (TERS). The appearance of the spin-waves only when the TERS tip is in close proximity to a nanocrystal edge suggests that the coupling of a localized plasmon with spinwaves arises due to broken symmetry at the nanoparticle border and the additional electric field confinement. Beyond phonon confinement effects previously reported in similar systems, this work offers significant insights on the plasmon-assisted generation and detection of spin-waves optically induced.

Received 25th February 2015, Accepted 27th April 2015

DOI: $10.1039 / \mathrm{c} 5 \mathrm{nr} 01277 \mathrm{e}$

www.rsc.org/nanoscale earth. Its nanoparticle form is especially interesting since particle shape and size can be easily controlled in sol-gel and chemical route synthesis. ${ }^{9-11}$ This control of particle size and shape, along with its high monodispersity, provide a model system for the study of size-dependent properties such as magnetism, optical absorption, and even in applications as an active electrode in lithium ion batteries. ${ }^{12-17}$ Details on magnetic properties and size dependence of iron oxide colloidal particles were reported earlier by the group of Matijevic. ${ }^{18,19}$ Hematite is a classic antiferromagnetic material below the Morin transition temperature of $260 \mathrm{~K}$. Above this temperature up to the Néel temperature of $960 \mathrm{~K}$ hematite shows a ferromagnetic behavior. This weak ferromagnetism is perceptible since the magnetic moments of the two sublattices are not quenched but produce a non-zero component normal to the basal plane. The particles become superparamagnetic if the size is reduced down to $8-41 \mathrm{~nm}$; some discrepancy in the precise critical size value for the onset of superparamagnetic behavior still exists. ${ }^{20,21}$ The exchange coupling between nanoparticles could also contribute to the suppression of superparamagnetism ${ }^{22}$ that might explain the different critical sizes reported.

In this work we report on the appearance of a band in the Raman spectra of iron oxide nanoparticles interacting with gold and silver tips that are used in tip-enhanced Raman spectroscopy. This band observed around $1584 \mathrm{~cm}^{-1}$ is due to the excitation of a two-magnon mode (spin-wave) produced by a 
phonon-mediated coupling of the spin of d-orbital electrons with strongly localized electromagnetic field in the vicinity of the tip apex. There were some controversies in the assignment of the magnon mode in hematite. The early work of Cardona et $a .^{23,24}$ supported a magnon mode position at around $1320 \mathrm{~cm}^{-1}$. Later on it was shown by McCarty ${ }^{25}$ that this mode did not involve any magnon scattering but was second order of a forbidden phonon mode. The assignment of this magnon mode is still under debate. ${ }^{26}$ The inelastic interaction of light with spin-waves played a major role in the discovery of giant magnetoresistance by Grünberg et al. in $1989 .^{27}$ A magnon or spin-wave refers to the collective oscillation of spin moments in a crystal. Several theoretical and experimental studies on spin-waves in metallic nanoparticles were recently reported ${ }^{28,29}$ as well as the role of electron-magnon interaction for tunneling transport ${ }^{30}$ and in the thermopower. $^{31}$ In addition, the control of optically induced spinwaves could lead to breakthroughs in spintronics by allowing the generation of spin-waves using spatially shaped light beams. ${ }^{32}$ The work we report here on hematite $\left(\alpha-\mathrm{Fe}_{2} \mathrm{O}_{3}\right)$ nanoparticles shows the generation of spin-waves mediated by metallic nanostructures in surface- and tip-enhanced Raman spectroscopy.

\section{Experimental}

\section{Synthesis of $\alpha-\mathrm{Fe}_{2} \mathrm{O}_{3}$ nanoparticles}

Hematite nanoparticles (NPs) were synthesized by forced hydrolysis of an acidic ferric chloride solution (final pH 2.0) at $95{ }^{\circ} \mathrm{C}$ as described by Jolivet et al. for ferric nitrate solutions. ${ }^{10}$ A stock solution of iron(III) precursor was obtained by mixing $10 \mathrm{~cm}^{3}$ of $\mathrm{FeCl}_{3}$ (3 mol L${ }^{-1}$ aqueous solution $45 \%$ Riedel-de Haën) and $60 \mathrm{~cm}^{3}$ of $\mathrm{HCl}\left(0.2 \mathrm{~mol} \mathrm{~L}^{-1}\right)$. All preparations were made using doubly ionized water, filtered with a $30000 \mathrm{Da}$ membrane. An amount of $7 \mathrm{~mL}$ of this stock solution was rapidly added under vigorous agitation into $300 \mathrm{~mL}$ of a hot $\mathrm{NaOH}$ solution $\left(10^{-2} \mathrm{~mol} \mathrm{~L}^{-1}\right.$ diluted from $0.1 \mathrm{~N} \mathrm{NaOH}$, Normadose, VWR) in order to obtain a $\mathrm{pH}$ of 2.0. $\mathrm{NaOH}$ solution was added drop by drop using an automatic cylinder driven by a Metrohm Combi Titerur 3D until the programmed $\mathrm{pH}$ value was reached. The final iron(III) concentration of the suspension was $10^{-3}$ mol L ${ }^{-1}$. The compound precipitated brownish from the solution. The mixture was then poured in a polypropylene bottle and aged at $95{ }^{\circ} \mathrm{C}$ for 7 days without stirring. After aging, a stable colloidal suspension of red color was obtained containing the hematite NPs.

Standard X-ray diffraction (XRD) measurements were carried out with $\mathrm{Cu}$ K-alpha radiation. The crystallinity and purity of dried samples deposited on a glass plate were verified by XRD patterns. The observed peaks all corresponded to the expected ones for the hematite structure (JCPDS 33-0664). ${ }^{33}$

\section{Fabrication of metallic tips}

Gold and silver tips were fabricated by electrochemical etching. The end of a silver microwire with $50 \mu \mathrm{m}$ diameter and purity $99.99 \%$ (Goodfellow, UK) was immersed in a $\mathrm{KNO}_{3}$ solution. $\mathrm{A}+3 \mathrm{~V}$ bias voltage was applied to an aluminum counter electrode with respect to the wire. A similar process was used for obtaining metallic gold tips starting from a gold microwire with $50 \mu \mathrm{m}$ diameter and purity $99.99 \%$ but in this case a saturated solution of $\mathrm{NaBr}$ was used as the etching medium.

A drop of the as-prepared hematite nanoparticle dispersion was placed in contact with the tips leaving a uniform distribution of nanoparticles on the tip surface as confirmed by SEM observations. Sharp tip formation and nanoparticle adsorption and stability were verified using a scanning electron microscope from FEI (Nova NanoSEM 200). The silver cantilever used in the AFM TERS experiments was fabricated following a previous work. ${ }^{34}$

\section{Atomic force microscopy}

Two atomic force microscope devices were used to characterize hematite nanoparticle size and shape: a Nanoscope Dimension 3100 (Veeco Instruments) and a 5420 AFM (Agilent Technologies). The tips used for AFM imaging were silicon tips provided by Nanosensors (Switzerland) with a radius of $10 \mathrm{~nm}$ and a half-cone angle of $10^{\circ}$. Both AFMs were operated in tapping mode. Silicon (111) with a native oxide layer was used as a substrate to immobilize the nanoparticles for AFM observations. Measurements and sample preparation were performed following ref. 35. A 10\% concentration of the original solution was obtained by diluting in ultrapure water (Milli-Q). A drop of the diluted solution was brought in contact with the silicon substrate and then rapidly removed with filter paper leaving a trace of well-dispersed nanoparticles. Without any further treatment the sample was used for the AFM measurements performed at room temperature and ambient conditions.

\section{Raman spectroscopy}

Micro-Raman experiments were performed in the backscattering geometry within the spectral range of $200-1700 \mathrm{~cm}^{-1}$ using the $514.5 \mathrm{~nm}$ line of an $\mathrm{Ar}^{+}$laser and the $632.8 \mathrm{~nm}$ line of a HeNe laser. The Raman spectrometer was a LabRam HR800 with an optical microscope Olympus BX-40. A 50× long working distance objective (N.A. 0.5) was used to illuminate the sample and to collect the Raman signal. The diffraction limited resolution was $\lambda /(2 \mathrm{~N} . \mathrm{A}$. $) \approx 515 \mathrm{~nm}$ at $\lambda=514.5 \mathrm{~nm}$. A liquid nitrogen-cooled back-illuminated charge-coupled device was used for the detection of the Raman signal using a diffraction grating of 600 lines per $\mathrm{mm}$, a spectral resolution of $4 \mathrm{~cm}^{-1}$, and an acquisition time of $5 \mathrm{~s}$. The laser power was limited to $0.5-2 \mathrm{~mW}$ in order to minimize heating of the sample. Nanoparticles were deposited on the same substrates used in AFM, i.e. silicon (111) with a native oxide layer. The nanoparticles dispersion was not diluted for Raman spectroscopy measurements but the as-prepared concentrated solution was used instead. The enhanced spectra were obtained after briefly placing a microdrop $(5 \mu \mathrm{L})$ hematite colloid in contact with a freshly etched gold or silver tip followed by sucking off the drop. In such a way a uniform distribution of isolated nano- 

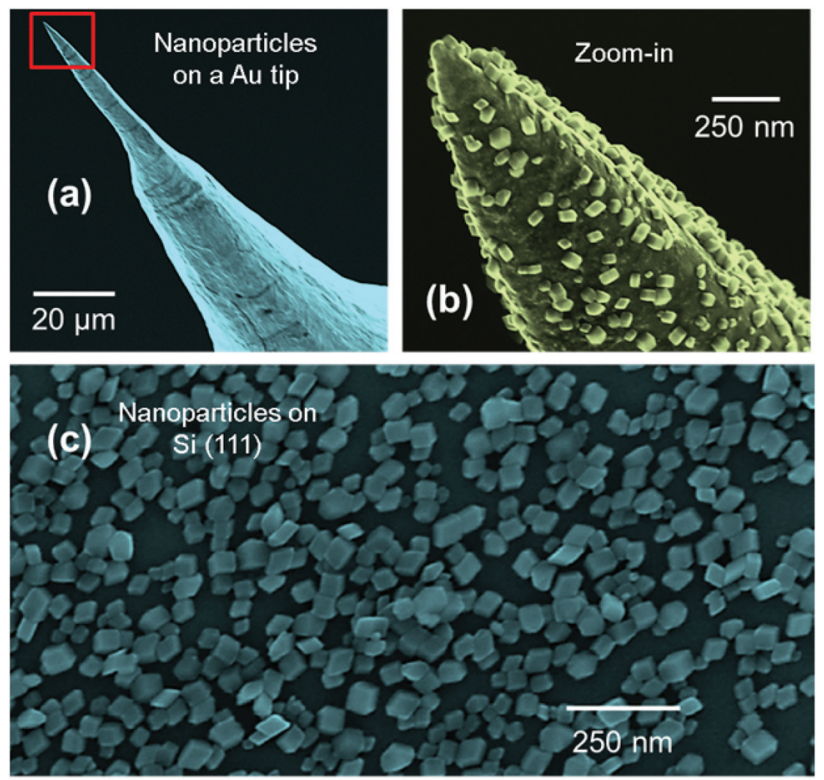

Fig. 1 Scanning electron microscopy imaging of the nanocrystals adsorbed on the two surfaces. (a) Nanoparticles deposited on a gold tip and (b) a zoom in at the tip apex observed after Raman measurements with laser irradiation of $\lambda=632.8 \mathrm{~nm}$ and power $1 \mathrm{~mW}$. (c) Nanocrystals deposited on an oxidised silicon (111) surface with no laser irradiation. Particle density appears to be smaller on gold tips as compared to the silicon surface. Note that there is no modification in particle morphology induced by the laser beam on the gold tip.

particles on the metallic tips was obtained as shown in Fig. 1 for the case of nanocrystals on a gold tip. The Raman signal of the bulk material was adapted from the RRUFF Project data (R040024) obtained using $532 \mathrm{~nm}$ excitation and $150 \mathrm{~mW}$ on a natural crystal from the University of Arizona Mineral Museum. ${ }^{36}$

\section{Tip-enhanced Raman spectroscopy}

TERS experiments were carried out with an AFM (Nanowizard II, JPK, Germany) mounted on an inverted microscope with confocal spectrometer (SP2750A, Acton Advanced, Princeton Instruments Roper Scientific, USA) and CCD camera (Pixis 400, Princeton Instruments Roper Scientific, USA). The HNPs were drop-coated on a thin glass microscope slide, and dried. The incident laser power was $1 \mathrm{~mW}$ at $532 \mathrm{~nm}$. For laser focusing an oil immersion objective (40×, N.A. 1.35, Olympus) was used. Spectra were recorded with an acquisition time of $5 \mathrm{~s}$.

\section{Results and discussion}

\section{Microscopic observations and surface-enhanced Raman spectroscopy}

In Fig. 1 the scanning electron microscopy (SEM) images display the hematite nanoparticles (NP) deposited on both substrates: on a gold tip and on a silicon (111) surface. Fig. 1a and $\mathrm{b}$ show nanoparticles adsorbed to the gold tip while in Fig. 1c the nanoparticles were deposited on the silicon surface.

Note that the nanocrystal density on the gold tip is considerably smaller than that on the silicon substrate. We concluded that any increase in Raman intensity for the metal tips cannot be due to a larger nanoparticle density but must be of plasmonic origin. Due to the strong electromagnetic field confinement at the tip, modifications of the nanocrystals during Raman measurements could be expected to affect spectra themselves. ${ }^{37}$ Such laser-induced effects could be ruled out by SEM measurements of nanoparticles before and after laser irradiation, as well as by spectroscopic signature of the HNPs. For comparison, the nanocrystals in Fig. 1c were not exposed to the laser beam while the nanoparticles on the tip (Fig. 1a and b) were used for Raman spectroscopy under $\lambda=$ $632.8 \mathrm{~nm}$ laser excitation and a power of $1 \mathrm{~mW}$ focused in the laser spot of $3 \mu \mathrm{m}^{2}$. Therefore, we assume that differences in the spectra, that will be discussed below do not originate from alterations of the samples during the enhanced Raman spectroscopy experiments. Since the high quality $\alpha-\mathrm{Fe}_{2} \mathrm{O}_{3}$ crystals possess high symmetry, only seven Raman active modes are predicted according to group theory. Four of these modes are observed in the spectral range of $200-450 \mathrm{~cm}^{-1} .^{26}$

In Fig. 2, Raman spectra of $\alpha-\mathrm{Fe}_{2} \mathrm{O}_{3}$ bulk crystal, nanoparticles deposited on Si (111), and nanoparticles adsorbed on a gold tip are compared. The similarity of the spectra demonstrates the crystallinity and unique phase and uniformity of the nanoparticles. Since additional details on the bulk sample are not available, the frequency shift differences between the HNPs and the bulk are not discussed further.

In the spectral region of $1200-1700 \mathrm{~cm}^{-1}$, two prominent bands can be observed in the enhanced spectra collected from nanoparticles on metallic tips (Fig. 3). The band centered around $1318 \mathrm{~cm}^{-1}$ is a second order 2LO phonon mode, while the one centered around $1584 \mathrm{~cm}^{-1}$ is attributed to a spinwave/two-magnon mode. ${ }^{38}$ Magnon modes in hematite were a subject of debate for some time and the 2LO phonon mode was originally attributed to a magnon mode. This assignment

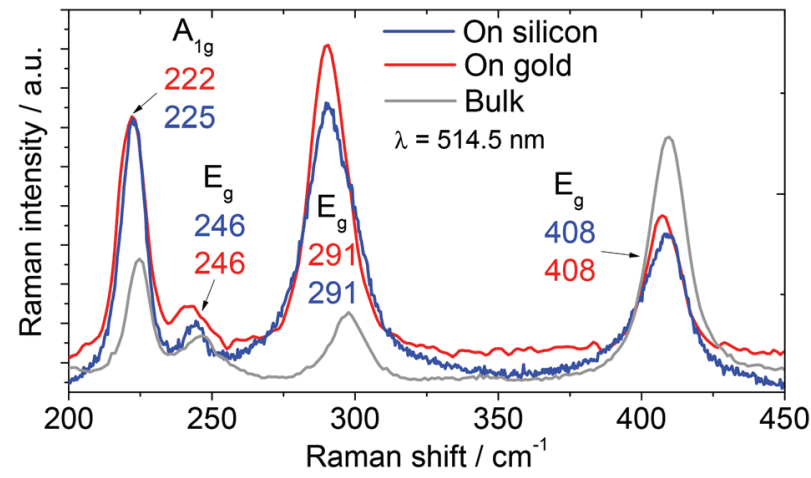

Fig. 2 Low frequency region of Raman spectra of hematite nanoparticles $(\lambda=514.5 \mathrm{~nm})$ and of a reference bulk substrate $(\lambda=532 \mathrm{~nm})$ taken from the RUFF database. ${ }^{36}$ 

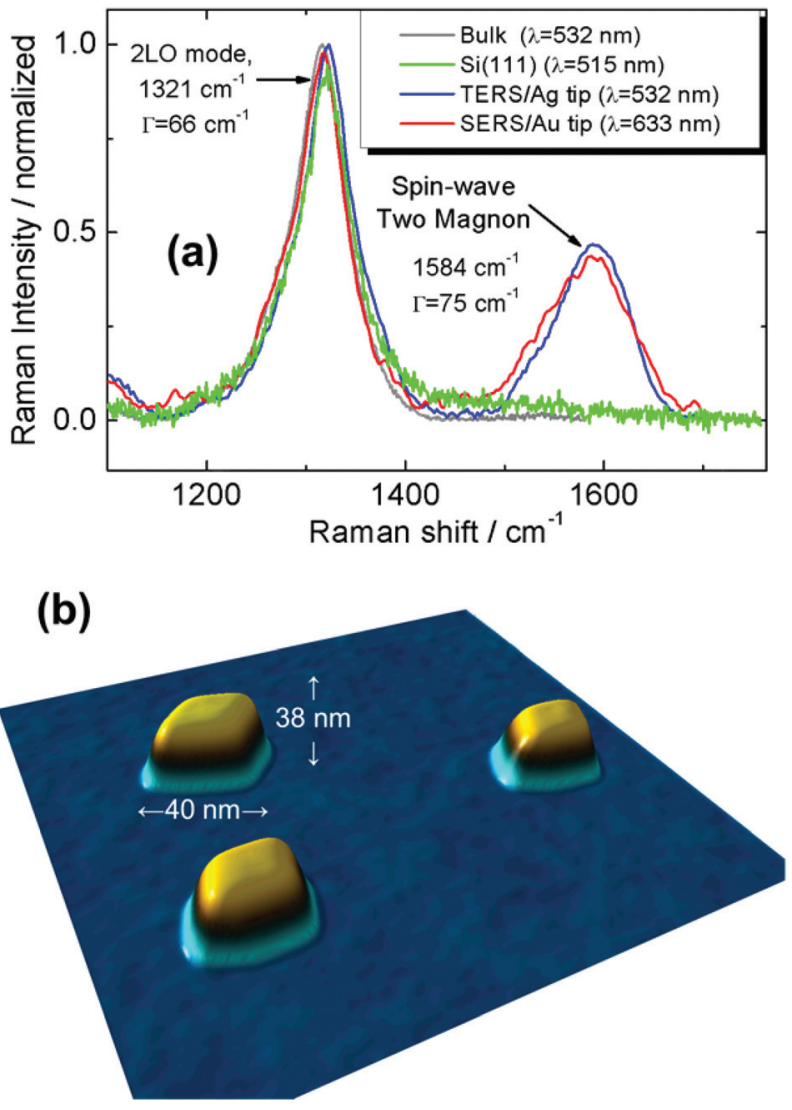

Fig. 3 (a) Second order 2LO mode and magnon mode visible in the Raman spectra. The spectrum of the bulk material measured at $\lambda=$ $532 \mathrm{~nm}$ was adapted from the RRUFF database. ${ }^{36}$ The spectra of nanoparticles on silicon using $514.5 \mathrm{~nm}$ excitation, and on a gold tip were measured using $632.8 \mathrm{~nm}$ excitation. The TERS spectrum of nanoparticles with a silver tip was measured under $532 \mathrm{~nm}$ excitation. (b) Atomic force microscopy image of the $\alpha-\mathrm{Fe}_{2} \mathrm{O}_{3}$ nanoparticles adsorbed on a silicon substrate. Their size is $(38 \pm 4) \mathrm{nm}$ with a rhombohedral shape made by the crystal planes of the $\{104\}$ family. ${ }^{35}$

was revised by McCarty. ${ }^{25}$ Recently, the shift and broadening of the magnon mode reported at around $1568 \mathrm{~cm}^{-1}$ in hematite nanoparticles was attributed to an effect of phonon confinement. ${ }^{39}$ Our observations are in agreement with the report of Owens et al. ${ }^{39}$ who found no change in the peak positions of the fundamental modes in nanoparticles of $(9 \pm 1) \mathrm{nm}$ size but reported a blue shift and broadening of the magnon mode attributed to the effect of phonon confinement. The difference in magnon mode peak position might be attributed to the difference in nanoparticle size between Owen's (5 nm) and this work due to the increase in lattice parameter by decreasing particle size as reported by Lu et al. ${ }^{40}$ Higher defect concentration causes downshift and broadening of the vibrational modes due to relaxation of selection rules. Owen's hematite particles were the product of laser induced phase transition from magnetite nanoparticles used as precursors, while our particles are well-defined nano-objects with high crystallinity. ${ }^{35}$ Thus, in addition to size effects, shape and defects can also account for the band shift observed between the two works.
From additional experiments, we did not find any significant difference in the results obtained under different excitation wavelength (green laser, $\lambda=514.5 \mathrm{~nm}$ ) and using a silver tip.

In Fig. 3b an AFM image of the nanoparticles deposited on silicon (111) is shown. The nanoparticles are monodispersed showing a rhombohedral shape defined by facets belonging to the crystal planes of the $\{104\}$ family. ${ }^{35}$ The nanoparticles have a size of $(38 \pm 4 \mathrm{~nm})$ determined from cross-section profiles made on the height AFM images. Such a size is too large for phonon confinement effects. In Raman spectra, phonon confinement is usually reflected as a red shift and broadening of the optical phonon modes for sizes less than $8 \mathrm{~nm},{ }^{41}$ which can be used to estimate the particle size. However, except for the $E_{\mathrm{g}}$ mode at $291 \mathrm{~cm}^{-1}$, such red shift was not observed.

An interesting result is shown in Fig. 3 regarding the band around $1584 \mathrm{~cm}^{-1}$ which is attributed to the spin-wave magnon mode. Note that this mode is not visible in the Raman spectra of nanoparticles deposited on silicon but it is clearly observed in the case of Raman spectra taken from nanoparticles adsorbed on metallic tips. This is the band that we will target in the following sections.

\section{Localized interaction of a Ag tip with nanoparticles in tip-enhanced Raman spectroscopy}

The capability to control the position of a metallic tip with nanoscale precision makes TERS a convenient method to carefully reproduce the detection of spin-waves. TERS spectra obtained in a line scan with a Ag tip are shown in Fig. 4a. The experiment was performed in tip-scan mode in a small region such, that the far-field contribution remained constant. Therefore, any spectral change can be attributed to the near-field generated at the $\mathrm{Ag}$ probe apex. The tip was scanned continuously over the glass substrate and the Raman intensity of all modes arose considerably when nanoparticles were in close proximity. This strong signal intensity increase is predominantly high for the spin-wave band around $1587 \mathrm{~cm}^{-1}$. Afterwards, the tip retraction results in a significant decrease in signal intensity of all modes. The band detected at $657 \mathrm{~cm}^{-1}$ is a LO mode (longitudinal optical) that is in general not Raman active; its appearance (although not yet clear) could be related to defects or relaxation of selection rules in hematite nanoparticles. ${ }^{42}$ The course of the selected bands along the line scan is given in the Raman intensity profile in Fig. $4 \mathrm{~b}$.

It is worth noticing that control experiments were performed on the tips before nanoparticle deposition in order to make sure that no carbon contamination contributes to the Raman spectra. ${ }^{43}$ Such control experiments are essential since the $\mathrm{D}$ and $\mathrm{G}$ bands of carbon and hydrocarbon species would overlap with the $2 \mathrm{LO}$ mode and spin-wave mode at $1584 \mathrm{~cm}^{-1}$ shown in Fig. 3. The electromagnetic field confined at the tipnanoparticle interface interacts with the optical phonons of the nanocrystals amplifying its signal. The striking appearance of the two-magnon mode in enhanced spectra can arise from the interaction of hematite nanoparticles with the metallic tip, and the influence of the electromagnetic field on 

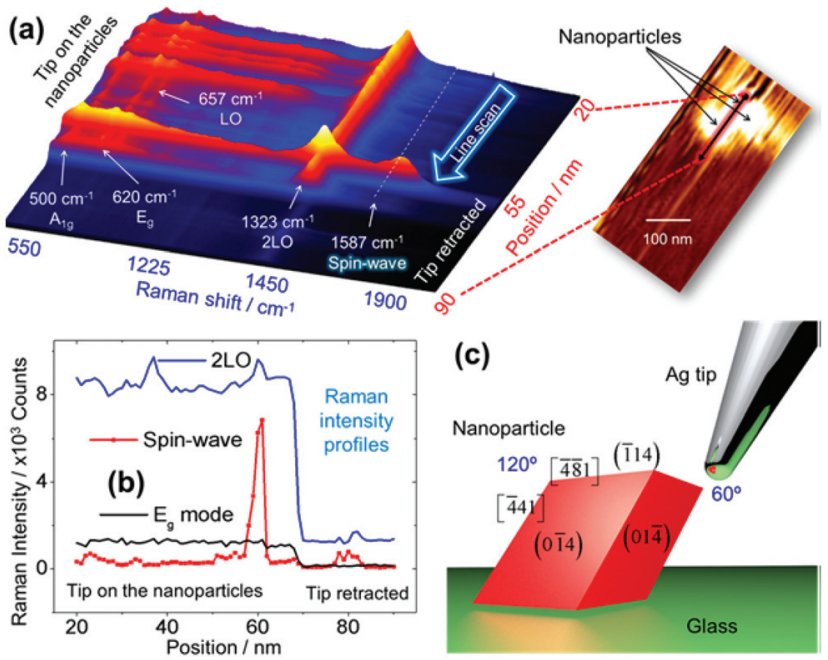

Fig. 4 (a) Tip-enhanced Raman spectra in a line scan along the nanoparticles/glass interface. The stacking representation shows several first order modes below $700 \mathrm{~cm}^{-1}$, the $2 \mathrm{LO}$ mode around $1323 \mathrm{~cm}^{-1}$ and the spin-wave mode around $1587 \mathrm{~cm}^{-1}$. The step size used to obtain this set of spectra was $1 \mathrm{~nm}$. In the inset is shown the AFM image acquired during the TERS line scan (b) intensity profiles of three modes along the line scan showing the sharp increase in the spin-wave intensity when the TERS tip is on the nanoparticle. This is followed by a decrease in the intensity of all modes when the tip was retracted (tip off). (c) A sketch of the tip-nanoparticle configuration shows the anisotropy between the two sides of the particle with respect to the tip. Crystallographic facets and edges are set according to ref. 35 .

the tip-nanoparticle interface, which determines the Raman signal intensity. Although the Raman intensity of all modes disappears at the moment the tip is retracted from the nanoparticles, a remarkable feature observed from the Raman intensity profile in Fig. $4 \mathrm{~b}$ is the non-uniform but sudden increase of the spin-wave mode. This puzzling and surprising result could be explained if we take into account the crystal structure and shape of these nanoparticles. In a previous study, the crystallinity and the morphology of hematite nanoparticles synthesized from hydrolysis were established to be of the family $\{104\}$ resulting in a rhombohedral shape. ${ }^{35}$

Such rhombohedral structure results in edges of the type [-4-81] and [841] delimiting the top and side facets that are equivalent (see ESI $\dagger$ ), but not equivalent to the edges of the opposite side of the nanoparticle that make a much sharper angle of $60^{\circ}$ between side facets $v s .120^{\circ}$ as shown in Fig. 4c. Even if for both edges the symmetry in the crystal is broken, the structural difference between the two kinds of edges contributes to the selective observation of the spin-waves at one of the sides of the particle but to a lower extend at the other. Moreover, since the electric field is much higher in those sharp regions due to the lightning rod effect, the light wave and spin-wave coupling is much more efficient in the sharp side of the particle, see sketch in Fig. 4c. The clarity of the AFM image acquired during the TERS line scan is not enough to discern between the two edge types (see inset in Fig. 4). Nevertheless, by considering the lighting rod effect it is reasonable to expect that the largest spin-wave enhancement occur at the nanoparticle edge with higher sharpness (with $60^{\circ}$ ). The high electric field confinement at the sharp nanoparticle edge is consistent with the work of Thomas et al. for other non-metallic systems. ${ }^{44}$ The TERS results shown in Fig. 4 could also help elucidate why we observe the spin-wave mode in the SERS experiments for which particles were adsorbed directly on silver and gold tips. Indeed, the roughness of the tips could also contribute to enhance the signal from the magnon-mode since a fraction of the crystals deposited with the sharp edges in direct contact with the metal, making an enhancement of the magnon mode possible due to close location of localized surface plasmons and edges supporting the spin-waves. However, a negative factor of the tip surface roughness is the attenuation of surface plasmon polariton compression that could play an important role in TERS as demonstrated by the work of Zaccaria et al. ${ }^{45}$

\section{The two-magnon mode band position and intensity}

As mentioned earlier, there were some conflicting reports about assignment and peak position of the two-magnon mode in $\alpha-\mathrm{Fe}_{2} \mathrm{O}_{3}$. There is a way to estimate the spin-wave peak position by determining its energy using the Ising model. The Hamiltonian function for the magnetic system can be written as: $H(S)=\sum_{i, j} J_{i, j} S_{i} \cdot S_{j}-\mu \sum_{j} H_{j} \cdot S_{j} \quad$ where $J_{i, j}$ denotes the exchange interaction between two neighboring $i$ and $j \mathrm{Fe}^{3+}$ ions, $S_{i}\left(\mathrm{Fe}^{3+}\right)=5 / 2$ is the spin of the ion. The last term defines the interaction weighted by the magnetic moment $\mu$ in the presence of an external magnetic field that in our case is zero, $H_{j}=0$.

We consider up to four near-neighbors in the [111] direction that represent the unit cell. Flipping a spin in the system at $T=0$ requires an energy given by:

$$
\begin{aligned}
\Delta E & =\sum_{n=1}^{4} J_{i} S z_{n} \sigma_{i} \\
\Delta E & =\frac{5}{2}\left[\begin{array}{l}
\left(-8.3 \mathrm{~cm}^{-1}\right) \cdot 1+\left(-2.2 \mathrm{~cm}^{-1}\right) \cdot 3 \cdot(-1) \\
+\left(41.3 \mathrm{~cm}^{-1}\right) \cdot 3+\left(32.3 \mathrm{~cm}^{-1}\right) \cdot 6
\end{array}\right]=789.5 \mathrm{~cm}^{-1}
\end{aligned}
$$

where $z_{n}$ is the number of neighbors at the $n$-th distance, and $\sigma_{i}$ is $+1(-1)$ if the spins are anti-parallel (parallel). The parameters $J_{i}, z_{n}, \sigma_{i}$ were adopted from the literature. ${ }^{46,47}$

This description implies the simultaneous creation of two magnons that are spatially very close to each other. Due to momentum conservation and the translational symmetry of the system each magnon has wave vectors $k$ and $-k$ (ref. 46) such that:

$$
E(-\vec{k})=E(\vec{k}) \Rightarrow E_{2 M}=2 E(\vec{k}) \Rightarrow \Delta E_{2 M}=1580 \mathrm{~cm}^{-1} .
$$

Therefore, the frequency of the two-magnon mode determined by the simple Ising model, neglecting magnonmagnon interaction, predicts a peak position in good agreement with our experimental observations. Note that taking into account the interaction between the two magnons would result in a decrease of the two-magnon energy calculated 
above. Ab initio calculations results recently reported by Erlebach et al. ${ }^{48}$ show that magnetic parameters of $\mathrm{Fe}_{2} \mathrm{O}_{3}$ clusters differ from bulk hematite, displaying strong antiferromagnetic interactions. These changes in the magnetic parameters result in the upshift of the spin-wave energy accounting for the higher value experimentally observed in the nanoparticles with respect to the Ising model calculations performed for the bulk material. In addition, experimental investigations are still needed to pinpoint the detailed mechanism behind the appearance of spin-waves in the enhanced Raman spectra. The AFM topography at the sharp edge is representative of the size and shape of the tip, but not the particle, due to tip selfimaging. ${ }^{49}$ Nevertheless, including high-resolution AFM imaging during TERS will be necessary in future experiments in order to unambiguously identify the crystallographic facet of the particle.

The intensity of the magnon mode, similar to that of higher orders modes, strongly dependents on resonance conditions. The resonance conditions are met if the photon energy of the Raman laser excitation matches an electronic transition. However, this effect alone cannot explain why the Raman intensity of the spin-wave is negligible for particles deposited on silicon with respect to particles in SERS and TERS experiments since for all cases the laser energy was the same. Besides the plasmonic contribution discussed in the previous section, an additional mechanism could take place that changes the resonance conditions leading to larger enhancement. Sá et $a l .{ }^{50}$ recently reported on the charge transfer between gold nanoparticles and $\mathrm{TiO}_{2}$ induced by localized surface plasmons. It is reasonable that charge transfer occurs due to the localized surface plasmons in SERS and TERS that shift the energy levels in the band structure of $\alpha-\mathrm{Fe}_{2} \mathrm{O}_{3}$. Such situation could result in the large increase of the magnon mode intensity observed here.

In addition to the spin-wave excitation at the nanoparticle edge, we should also consider the physical mechanisms for the selective high spatial confinement and activation of this mode. Deng et al. ${ }^{51}$ showed that regions with local symmetry breaking in a plasmonic waveguide promote the coupling with the far field and launch surface plasmons in a metallic wire. In our case, it is the plasmon wave vector of the TERS tip that should match the wave vector of the spin-wave. Besides the phase-matching, the energy between the plasmon $(\mathrm{eV})$ and the spin-wave (meV) need to be in resonance. The local symmetry breaking at the nanoparticle edge could help to compensate this mismatch launching spin-waves at the edge. Moreover, as is well known in SERS, a coupled system such as the nanoparticle-tip illuminated under resonance conditions would create a highly confined electric field region, the so-called hotspot. ${ }^{52}$ These two mechanisms are not mutually exclusive. The higher localization and intensity of electric field at the nanoparticle edge and the strong electric field gradient that satisfy the phase-matching conditions would contribute to the coupling and activation of the spin-wave. These scenarios could be verified in future works by calculation of the dispersion relation for the spin-wave and plasmon system, spin-wave dynamics at the single nanoparticle level, and by simulations of the spatial distribution of electric field enhancement around the particle-tip nanogap.

\section{Conclusions}

In this contribution surface- and tip-enhanced Raman experiments of iron oxide nanocrystals (hematite) adsorbed on different substrates were presented. The enhanced Raman spectra obtained by the deposition of $\alpha-\mathrm{Fe}_{2} \mathrm{O}_{3}$ nanoparticles on noble metallic tips yielded a band around $1584 \mathrm{~cm}^{-1}$ assigned to a spin-wave (magnon) mode. This band is practically absent in conventional Raman spectra of those nanoparticles directly deposited on silicon (111) or on a glass substrate. The findings reported here show the coupling of localized optical fields and spin-waves. Since localized surface plasmons can provide a way to excite and control spin-waves this work has potential implications in spintronics and magnonics.

\section{Acknowledgements}

We thank Alexander Villabona for assistance with the SERS experiments, Susanne Hartmann and Jana Kalbacova for valuable feedback on the manuscript. This work was supported by the following projects: DFG Research Unit 1713 "Sensoric Micro- and Nanosystems". DFG project ZA146/22-1 "Raman investigations of $\mathrm{In}(\mathrm{Ga}) \mathrm{As} / \mathrm{Al}(\mathrm{Ga}) \mathrm{As}$ self-assembled quantum dot structures: from ensembles to single quantum dots". Cluster of Excellence "Center for Advancing Electronics Dresden” cfaed. VD and TDG acknowledge financial support from the State of Thuringia (FKZ: 2011 FE 9048; 2011 VF 0016) and the Deutsche Forschungsgemeinschaft (DE 1376/5-1). This work was performed in the context of the European COST Action MP1302 Nanospectroscopy.

\section{References}

1 C. Chen, N. Hayazawa and S. Kawata, Nat. Commun., 2014, 5,3312 .

2 T. Deckert-Gaudig and V. Deckert, Curr. Opin. Chem. Biol., 2011, 15, 719-724.

3 D. Zhang, U. Heinemeyer, C. Stanciu, M. Sackrow, K. Braun, L. E. Hennemann, X. Wang, R. Scholz, F. Schreiber and A. J. Meixner, Phys. Rev. Lett., 2010, 104, 056601.

4 M. D. Sonntag, J. M. Klingsporn, L. K. Garibay, J. M. Roberts, J. A. Dieringer, T. Seideman, K. A. Scheidt, L. Jensen, G. C. Schatz and R. P. Van Duyne, J. Phys. Chem. C, 2011, 116, 478-483.

5 R. Zhang, Y. Zhang, Z. C. Dong, S. Jiang, C. Zhang, L. G. Chen, L. Zhang, Y. Liao, J. Aizpurua, Y. Luo, J. L. Yang and J. G. Hou, Nature, 2013, 498, 82-86.

6 D. A. Schmidt, I. Kopf and E. Bruendermann, Laser Photonics Rev., 2012, 6, 296-332. 
7 C. Georgi and A. Hartschuh, Appl. Phys. Lett., 2010, 97, 143117.

8 X. Qian, X.-H. Peng, D. O. Ansari, Q. Yin-Goen, G. Z. Chen, D. M. Shin, L. Yang, A. N. Young, M. D. Wang and S. Nie, Nat. Biotechnol., 2008, 26, 83-90.

9 T. Sugimoto, Y. S. Wang, H. Itoh and A. Muramatsu, Colloids Surf., A, 1998, 134, 265-279.

10 J. P. Jolivet, C. Chaneac and E. Tronc, Chem. Commun., 2004, 481-487, DOI: 10.1039/b304532n.

11 K. Supattarasakda, K. Petcharoen, T. Permpool, A. Sirivat and W. Lerdwijitjarud, Powder Technol., 2013, 249, 353-359.

12 F. Bodker and S. Morup, Europhys. Lett., 2000, 52, 217-223.

13 Y. P. He, Y. M. Miao, C. R. Li, S. Q. Wang, L. Cao, S. S. Xie, G. Z. Yang, B. S. Zou and C. Burda, Phys. Rev. B: Condens. Matter, 2005, 71, 125411.

14 C. Wu, P. Yin, X. Zhu, C. OuYang and Y. Xie, J. Phys. Chem. B, 2006, 110, 17806-17812.

15 H. Cao, G. Wang, L. Zhang, Y. Liang, S. Zhang and X. Zhang, ChemPhysChem, 2006, 7, 1897-1901.

16 J. Wang, W. B. White and J. H. Adair, J. Am. Ceram. Soc., 2005, 88, 3449-3454.

17 J. Ma, X. D. Zhang, K. Z. Chen, G. C. Li and X. D. Han, J. Mater. Chem. A, 2013, 1, 5545-5553.

18 G. J. Muench, S. Arajs and E. Matijević, Phys. Status Solidi A, 1985, 92, 187-192.

19 N. Amin, S. Arajs and E. Matijevic, Phys. Status Solidi A, 1987, 104, K65-K68.

20 N. Amin and S. Arajs, Phys. Rev. B: Condens. Matter, 1987, 35, 4810-4811.

21 T. P. Raming, A. J. A. Winnubst, C. M. van Kats and A. P. Philipse, J. Colloid Interface Sci., 2002, 249, 346-350.

22 C. Frandsen, C. R. H. Bahl, B. Lebech, K. Lefmann, L. T. Kuhn, L. Keller, N. H. Andersen, M. von Zimmermann, E. Johnson, S. N. Klausen and S. Morup, Phys. Rev. B: Condens. Matter, 2005, 72, 214406.

23 R. Merlin, T. P. Martin, A. Polian, M. Cardona, B. Andlauer and D. Tannhauser, J. Magn. Magn. Mater., 1978, 9, 83-85.

24 T. P. Martin, R. Merlin, D. R. Huffman and M. Cardona, Solid State Commun., 1977, 22, 565-567.

25 K. F. McCarty, Solid State Commun., 1988, 68, 799-802.

26 D. L. A. deFaria, S. V. Silva and M. T. deOliveira, J. Raman Spectrosc., 1997, 28, 873-878.

27 G. Binasch, P. Grünberg, F. Saurenbach and W. Zinn, Phys. Rev. B: Condens. Matter, 1989, 39, 4828-4830.

28 C. M. Canali and A. H. MacDonald, Phys. Rev. Lett., 2000, 85, 5623-5626.

29 J. von Delft and D. C. Ralph, Phys. Rep., 2001, 345, 61173.

30 Ł. Michalak, C. M. Canali and V. G. Benza, Phys. Rev. Lett., 2006, 97, 096804.

31 E. McCann and V. I. Fal'ko, Phys. Rev. B: Condens. Matter, 2003, 68, 172404.
32 T. Satoh, Y. Terui, R. Moriya, B. A. Ivanov, K. Ando, E. Saitoh, T. Shimura and K. Kuroda, Nat. Photonics, 2012, 6, 662-666.

33 P. D. File, Powder Diffraction, International Centre for Diffraction Data, Swarthmore, PA 19081, 2000.

34 R. Rodriguez, E. Sheremet, S. Müller, O. Gordan, A. Villabona, S. Schulze, M. Hietschold and D. Zahn, Rev. Sci. Instrum., 2012, 83, 123708.

35 R. D. Rodriguez, D. Demaille, E. Lacaze, J. Jupille, C. Chaneac and J.-P. Jolivet, J. Phys. Chem. C, 2007, 111, 16866-16870.

36 R. Downs, In: The RRUFF Project: an integrated study of the chemistry, crystallography, Raman and infrared spectroscopy of minerals, Program and abstracts of the 19th general meeting of the international mineralogical association in Kobe, Japan, O03-13, 2006.

37 A. Ahlawat and V. G. Sathe, J. Raman Spectrosc., 2011, 42, 1087-1094.

38 S. Azuma, M. Sato, Y. Fujimaki, S. Uchida, Y. Tanabe and E. Hanamura, Phys. Rev. B: Condens. Matter, 2005, 71, 014429.

39 F. J. Owens and J. Orosz, Solid State Commun., 2006, 138, 95-98.

40 L. Lu, L. Li, X. Wang and G. Li, J. Phys. Chem. B, 2005, 109, 17151-17156.

41 M. N. Islam and S. Kumar, Appl. Phys. Lett., 2001, 78, 715717.

42 D. L. A. de Faria and F. N. Lopes, Vib. Spectrosc., 2007, 45, 117-121.

43 J. Kalbacova, R. D. Rodriguez, V. Desale, M. Schneider, I. Amin, R. Jordan and D. R. Zahn, Nanospectroscopy, 2014, 1, 12-18.

44 S. Thomas, M. Krueger, M. Foerster, M. Schenk and P. Hommelhoff, Nano Lett., 2013, 13, 4790-4794.

45 R. P. Zaccaria, F. De Angelis, A. Toma, L. Razzari, A. Alabastri, G. Das, C. Liberale and E. Di Fabrizio, Opt. Lett., 2012, 37, 545-547.

46 R. J. Elliott and M. F. Thorpe, J. Phys. C: Solid State Phys., 1969, 2, 1630-1643.

47 M. J. Massey, U. Baier, R. Merlin and W. H. Weber, Phys. Rev. B: Condens. Matter, 1990, 41, 7822-7827.

48 A. Erlebach, C. Huhn, R. Jana and M. Sierka, Phys. Chem. Chem. Phys., 2014, 16, 26421-26426.

49 R. D. Rodriguez, E. Lacaze and J. Jupille, Ultramicroscopy, 2012, 121, 25-30.

50 J. Sa, G. Tagliabue, P. Friedli, J. Szlachetko, M. H. RittmannFrank, F. G. Santomauro, C. J. Milne and H. Sigg, Energy Environ. Sci., 2013, 6, 3584-3588.

51 P. Deng, W. Hong and X. Hong-Xing, Chin. Phys. B, 2013, 22, 097305.

52 S. L. Kleinman, R. R. Frontiera, A. I. Henry, J. A. Dieringer and R. P. Van Duyne, Phys. Chem. Chem. Phys., 2013, 15, 21-36. 